\title{
Real-life feasibility and effectiveness of home-based pulmonary rehabilitation in chronic obstructive pulmonary disease requiring medical equipment
}

This article was published in the following Dove Press journal: International Journal of COPD

\author{
Jérémy B Coquart ${ }^{1}$ \\ Olivier Le Rouzic ${ }^{2}$ \\ Ghazi Racil ${ }^{3}$ \\ Benoit Wallaert ${ }^{2}$ \\ Jean-Marie Grosbois ${ }^{4}$ \\ 'CETAPS, EA 3832, UFR STAPS, \\ University of Rouen, Normandie- \\ Univ, Mont Saint Aignan, France; \\ ${ }^{2}$ Department of Respiratory \\ Diseases, University of Lille, CHRU \\ Lille, Lille, France; ${ }^{3}$ Department of \\ Biology, Faculty of Sciences, El Manar \\ University, Tunis, Tunisia; ${ }^{4}$ FormAction \\ Santé, Pérenchies, France
}

Correspondence: Jérémy B Coquart EA 3832, UFR STAPS, CETAPS,

Université de Rouen, Boulevard Siegfried, F-7682I Mont Saint Aignan

Cedex, France

Tel +33235 I46775

Email jeremy.coquart@univ-rouen.fr
Background: Pulmonary rehabilitation (PR) is a key treatment of chronic obstructive pulmonary disease (COPD) but studies are still needed to identify the most pertinent criteria to personalize this intervention and improve its efficacy.

Objective: This real-life retrospective study compared the effects of home-based PR on exercise tolerance, anxiety, depression, and health-related quality of life (HRQoL) in COPD patients, according to their medical equipment.

Methods: Exercise tolerance, anxiety, depression, and HRQoL were evaluated in 109 patients equipped with long-term oxygen therapy (LTOT), 84 patients with noninvasive ventilation (NIV), 25 patients with continuous positive airway pressure (CPAP), and 80 patients with no equipment (NE), before, just after, and 6 and 12 months after PR.

Results: At baseline, the body mass index in the CPAP and NIV groups was higher $(p<0.05)$ than in the other two groups, and the forced expiratory volume in 1 second was lower in the LTOT and NIV groups $(p<0.001)$. All parameters improved after PR in the four groups $(p<0.05)$, but for exercise tolerance, only the 6-minute stepper test showed maintained improvement after 6 and 12 months, whereas the 10 times sit-to-stand and timed up-and-go tests were only improved just after PR. At every time point, exercise tolerance was lower in the LTOT group $(p<0.05)$, with a similar trend in the NIV group.

Conclusion: Despite differences in the medical equipment to treat COPD, home-based PR showed comparable feasibility, safety, and efficacy in all equipment-based groups. Medical equipment should therefore not be a barrier to home-based PR.

Keywords: personalized medicine, noninvasive ventilation, long-term oxygen therapy, continuous positive airway pressure

\section{Introduction}

Chronic obstructive pulmonary disease (COPD) is associated with progressive dyspnea and reduced exercise tolerance, both of which encourage sedentary lifestyles and considerably deteriorate health-related quality of life (HRQoL). ${ }^{1}$ Pulmonary rehabilitation (PR), a comprehensive yet personalized intervention including physical activities, health education, and psychological support, has been shown to be beneficial, regardless of age, sex, severity of airflow limitation or place where it is performed (hospital, outpatient clinic, or home). ${ }^{2}$ Indeed, recent studies have confirmed that home-based PR is safe and effective. ${ }^{3}$

Several types of medical equipment are often used to treat COPD patients with poor clinical status: long-term oxygen therapy (LTOT) is recommended for patients with severe hypoxemia, noninvasive ventilation (NIV) for patients with severe chronic 
hypercapnia and a history of hospitalization for acute respiratory failure, and continuous positive airway pressure (CPAP) for people with obstructive sleep apnea syndrome (OSAS) with or without COPD. ${ }^{4,5}$ Therefore, most COPD patients with airflow limitation are equipped with LTOT, NIV, or CPAP in their everyday lives.

Home-based PR for COPD patients requiring LTOT is effective in reducing mortality, exacerbation rate, intensive care unit admissions, emergency department visits, and ordinary hospital admissions. ${ }^{6}$ Moreover, very severe COPD patients treated with LTOT exhibit significantly greater improvements in dyspnea, exercise tolerance, HRQoL, and lung function than patients treated without LTOT after only 3 weeks of PR. ${ }^{7}$ In addition, PR associated with nocturnal NIV significantly enhances exercise tolerance, HRQoL, and lung function in patients with advanced COPD. ${ }^{8}$ Last, it has been demonstrated that nocturnal CPAP improves exercise tolerance in COPD patients with OSAS, but it is not known whether the benefits of long-term PR are increased with CPAP. ${ }^{9}$ Essentially, despite the call to investigate other long-term treatment effects during $\mathrm{PR},{ }^{10}$ it is still unknown whether LTOT, NIV, or CPAP have different impacts on PR benefits.

We hypothesized that home-based PR would be feasible and effective in improving COPD patients in the short and long terms regardless of the equipment prescribed to treat the disease. Therefore, this real-life retrospective observational study compared improvements in exercise tolerance, anxiety/depression, and HRQoL in COPD patients according to their medical equipment (ie, LTOT, NIV, and CPAP) immediately after a home-based PR program and then 6 and 12 months later.

\section{Methods}

\section{Patients}

This 5-year retrospective study included consecutive COPD patients who were following a home-based PR program. COPD diagnosis was confirmed in each patient before PR by persistent airflow limitation (post-bronchodilator forced expiratory volume in 1 second $\left[\mathrm{FEV}_{1}\right] /$ forced vital capacity $[$ FVC] $<0.70) .{ }^{4}$ COPD patients were excluded if they had mild airflow limitation (post-bronchodilator $\mathrm{FEV}_{1} \geq 80 \%$ predicted), dementia or poorly controlled psychiatric illness, neurological sequelae, or bone and joint diseases preventing physical activity. Patients were classified into LTOT, NIV, CPAP, or no equipment (NE) groups according to their medical equipment. The observational research protocol evaluation committee of the French Language Society of Pulmonology (CEPRO 2011-036) approved the analysis of the data collected from these patients, and written informed consent was obtained from each participant.

\section{Home-based PR}

Briefly, as described elsewhere, this individual home-based PR program consisted of 90-minute sessions once a week for 8 weeks, based on educational needs assessment. ${ }^{11,12}$ It comprised endurance exercises and resumption of the physical activities of daily living, therapeutic education, psychosocial support, and motivational communication to encourage health behavior changes and self-management. Each weekly session was conducted under the direct supervision of a team member. Patients were expected to continue performing the endurance exercises on their own the other days of the week, according to a personalized action plan.

Initially, individual endurance exercise on a cycle ergometer was performed in sequences of 10 minutes or less (if the patient was unable to perform this), at least 5 days per week, with the goal of reaching 30-45 minutes/day, in one or several sessions. ${ }^{11,12}$ The exercise intensity was prescribed on the basis of dyspnea symptoms (scores of 3-4 on the Borg scale) $)^{13}$ or effort perception (scores of 11-13 on the ratings of perceived exertion scale) ${ }^{14}$ to ensure that the effort would be perceived as moderate and that the patients would be able to maintain this exercise intensity in real life. ${ }^{2}$ The patients were encouraged to increase the durations of their daily living activities. In addition, three muscle strengthening exercises for the upper and lower limbs were systematically proposed (with instructions), lasting 10-15 minutes/day and using weights and dumbbells and/or elastic bands. Each exercise comprised a series of 10 repeated movements. A 1-minute recovery period was observed between exercises. Warm-up and stretching exercises were also recommended together with balance exercises whenever necessary.

\section{Accident protocol}

The PR agreement given to the patients before PR included an accident protocol. An accident was defined as death, hospitalization, or emergency care required for heart or orthopedic incidents during the 8 weeks of PR. The patient and/or the physiotherapist could declare the occurrence of an accident. Patients were asked to interrupt all physical activities in the case of any abnormal sensations, especially chest or joint pain, and to contact both the rehabilitation team and the attending physician.

\section{Assessments}

Patients were evaluated at home just before and after the intervention period and 6 and 12 months after the end of PR. 
The evaluation of exercise tolerance included a 10 times sit-to-stand test (10STS), a timed up-and-go test (TUG) and a 6-minute stepper test (6MST). ${ }^{15-17}$ The 10STS consists of standing up until reaching full knee extension and sitting back down 10 times as fast as possible while seated at the front of a 42-cm high chair. ${ }^{15}$ During the TUG, patients stand up from a standard armchair, walk $3 \mathrm{~m}$, turn, walk back, and sit down again as quickly as possible. ${ }^{16}$ The time to complete the TUG is recorded with a minimal clinically important difference (MCID) of 1.5 seconds. Last, the number of steps taken in 6 minutes was collected during a 6MST as previously described. ${ }^{18}$ The stepper (Go Sport ${ }^{\circledR}$, Grenoble, France), with a step height fixed at $20 \mathrm{~cm}$, was placed near a wall to support patients if they became unbalanced or exhausted. The patients could freely regulate their stepping rate to reach the highest number of strokes. 6MST MCID has been estimated at 40 strokes (if one stroke corresponds to a half cycle: up or down). ${ }^{19}$

Psychological status was assessed by the Hospital Anxiety and Depression (HAD) scale, and HRQoL was assessed by the Maugeri Foundation Respiratory Failure Questionnaire (MRF-28), the Visual Simplified Respiratory Questionnaire (VSRQ), and the COPD-specific HRQoL Questionnaire (VQ11). ${ }^{20-23}$ The HAD scale determined the patient's psychological state in terms of anxiety and depression. ${ }^{20}$ This questionnaire has 14 items (seven items for each psychological state), with responses scored on a scale of $0-3$ to indicate symptom frequency (the higher the frequency, the higher the score is). The MCID for both scores (ie, anxiety and depression) is a change of 1.5 units or greater in magnitude. ${ }^{24}$ The MRF-28 is composed of 28 items covering three theoretical components (daily activity, cognitive function, and invalidity) and gives a final score ranging from 0 to 100 , with higher scores reflecting a higher degree of impairment. ${ }^{21}$ The MCID of the MRF-28 is unknown. The VSRQ is a valid and reliable questionnaire to assess HRQoL especially developed for patients with COPD. ${ }^{22}$ It comprises eight visual analog scales ranging from 0 to 10 , with a total score ranging from 0 to 80 . A higher score indicates better HRQoL. The MCID of the VSRQ is equal to 3.4 units. Last, the VQ11 comprises 11 items distributed across three components (functional: three items, psychological: four items, and social: four items) and a top level combining these three components. ${ }^{23}$ A higher score indicates less favorable quality of life. The MCID is unknown.

\section{Statistical analysis}

Data are reported as mean and standard deviation. For all data, normal Gaussian distributions were verified by the Shapiro-Wilk test and homogeneity of variance by the Levene's test. When the data did not pass the test for normality and/or homogeneity of variance, they were log transformed. For initial (before PR) data, a general linear model with a one-way design was used to compare the groups (ie, LTOT, NIV, CPAP, and NE). If significant differences were obtained, a Bonferroni post hoc test was conducted.

Possible effects of the rehabilitation period were tested from the general linear model (groups: LTOT, NIV, CPAP, and NE) for repeated measures (before and after the 2-, 6-, and 12-month intervention periods). The sphericity was checked by the Mauchly's test, and when it was not met, the significance of $F$-ratios was adjusted according to the Greenhouse-Geisser procedure (epsilon correction factor $<0.75$ ) or the Huynh-Feldt procedure (epsilon correction factor $\geq 0.75$ ). When significant differences were obtained, a Bonferroni post hoc test was conducted. For categorical data about the number of responders, the frequencies were compared using Pearson's chi-square test.

Statistical significance was set at $p<0.05$, and all analyses were performed with the IBM Statistical Package for the Social Sciences (version 18.0; IBM Corporation, Armonk, NY, USA).

\section{Results}

A total of 298 patients were included (Figure 1). They were mainly men older than 60 years with slight overweight and severe to very severe airflow limitation (Table 1). Few patients were classified into the CPAP group (8.4\%), whereas $36.6 \%$ were classified into the LTOT group, $28.2 \%$ into the NIV group, and $26.8 \%$ into the NE group. Only 19 patients did not complete the 8-week PR program but none for accidents or cardiovascular or orthopedic incidents due to exercise conditioning (Figure 1). Ultimately, 83.6\% and $76.5 \%$ of the patients were evaluated after 6 and 12 months, respectively.

At baseline (Table 1), body mass index (BMI) was significantly higher in the CPAP and NIV groups than in the

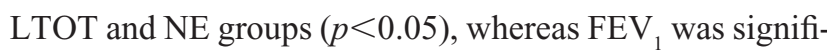
cantly lower in the LTOT and NIV groups compared with the two other groups $(p<0.001)$. We found that $20 \%, 38 \%$, $80 \%$, and $18 \%$ of the patients in the LTOT, NIV, CPAP, and NE groups were obese, respectively. Furthermore, three or more comorbidities were found for $76 \%, 82 \%, 88 \%$, and $70 \%$ patients in the LTOT, NIV, CPAP, and NE groups, respectively. At baseline and the other time points, LTOT patients exhibited significantly lower exercise tolerance, with the same trend for the NIV group, whereas psychological and HRQoL evaluations did not differ across the four groups (Table 2). 
PR programs performed $(n=298)$

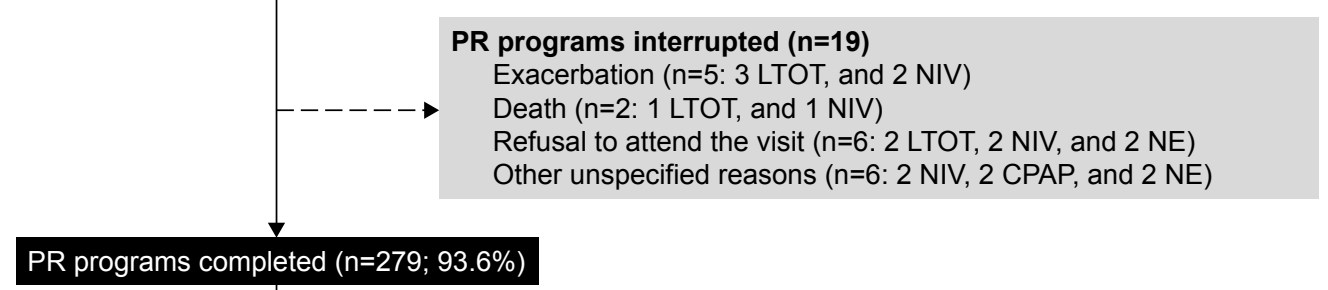

Patients not evaluated at 6 months $(n=30)$

Exacerbation $(n=1: 1$ LTOT)

Death ( $n=14: 5$ LTOT, 7 NIV, 1 CPAP, and 1 NE)

Refusal to attend the visit ( $n=6: 3$ LTOT, 1 NIV, 1 CPAP, and 1 NE)

Loss to follow-up ( $n=6: 1 \mathrm{NIV}$, and $5 \mathrm{NE}$ )

Lung transplantation ( $n=3: 3$ LTOT)

Evaluation at 6 months $(n=249 ; 83.6 \%)$

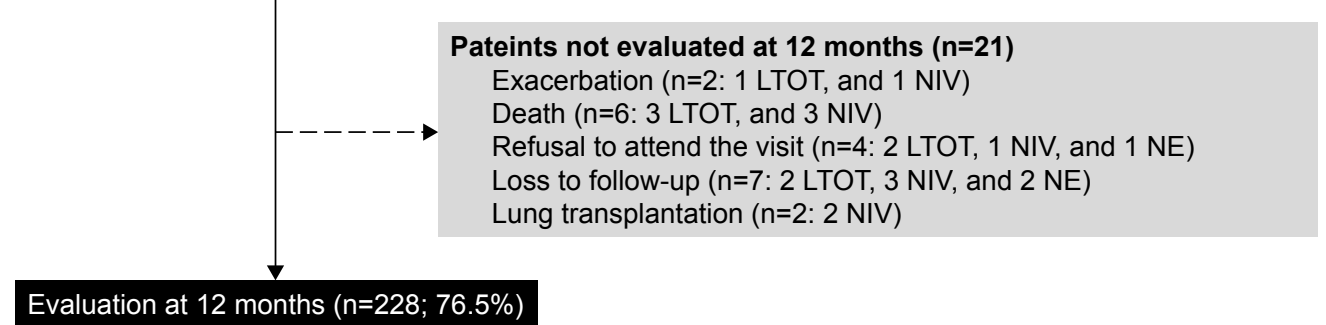

Figure I Flow diagram of patients with chronic obstructive pulmonary disease managed by home-based PR.

Abbreviations: CPAP, continuous positive airway pressure; LTOT, long-term oxygen therapy; NE, no equipment; NIV, noninvasive ventilation; PR, pulmonary rehabilitation.

In all groups, all parameters of exercise tolerance, psychological status, and HRQoL were significantly improved just after PR ( $p<0.05$; Table 2$)$. Interestingly, this improvement was always significant after 6 and 12 months in all groups for 6MST, psychological status, and HRQoL scores but not for 10STS and TUG $(p<0.01)$. Last, the percentages of patients who improved their parameters above the MCID were comparable across the four groups except for 6MST,

Table I Baseline characteristics of patients according to their medical equipment

\begin{tabular}{|c|c|c|c|c|c|}
\hline Characteristics & $\begin{array}{l}\text { Total } \\
\text { patients }\end{array}$ & LTOT & NIV & CPAP & NE \\
\hline Sample size $(n)$ & 298 & 109 & 84 & 25 & 80 \\
\hline Men to women ratio $(n)$ & $199 / 99$ & $73 / 36$ & $56 / 28$ & $20 / 5$ & $50 / 30$ \\
\hline Age (years) & $63.8 \pm 11.0$ & $64.8 \pm 11.8$ & $64.9 \pm 8.8$ & $64.9 \pm 11.9$ & $60.7 \pm 11.2$ \\
\hline BMI $\left(\mathrm{kg} / \mathrm{m}^{2}\right)$ & $27.0 \pm 7.6$ & $24.9 \pm 6.5^{b}$ & $29.4 \pm 9.0^{c}$ & $33.6 \pm 5.5^{c}$ & $25.6 \pm 6.3^{b}$ \\
\hline $\mathrm{FEV}_{1}(\mathrm{~L})$ & $1.117 \pm 0.536$ & $0.930 \pm 0.423^{d}$ & $0.962 \pm 0.464^{\mathrm{d}}$ & $1.409 \pm 0.575$ & $1.380 \pm 0.563$ \\
\hline $\mathrm{FEV}_{1}(\%$ theoretical value) & $40.9 \pm 17.1$ & $35.0 \pm 14.9^{d}$ & $34.3 \pm 14.5^{\mathrm{d}}$ & $53.0 \pm 16.1$ & $49.9 \pm 16.1$ \\
\hline FVC (\% theoretical value) & $66.2 \pm 20.1$ & $59.9 \pm 19.8^{d}$ & $60.6 \pm 18.4^{a}$ & $74.7 \pm 16.6$ & $75.8 \pm 18.5$ \\
\hline $\mathrm{FEV}_{\perp} / \mathrm{FVC}$ & $50.6 \pm 12.8$ & $46.2 \pm 11.7^{\mathrm{d}}$ & $48.6 \pm 12.4^{e}$ & $58.3 \pm 9.1$ & $54.9 \pm 12.9$ \\
\hline Moderate airflow limitation $\left(50 \% \leq \mathrm{FEV}_{1}<80 \%\right), \mathrm{n}(\%)$ & $71(23.8)$ & $15(13.8)^{d}$ & $10(11.9)^{d}$ & $12(48.0)$ & $34(42.5)$ \\
\hline Severe airflow limitation $\left(30 \% \leq \mathrm{FEV}_{1}<50 \%\right), \mathrm{n}(\%)$ & $132(44.3)$ & $48(44.0)$ & $35(4 I .7)$ & II (44.0) & $38(47.5)$ \\
\hline Very severe airflow limitation $\left(\mathrm{FEV}_{1}<30 \%\right), \mathrm{n}(\%)$ & $95(31.9)$ & $46(42.2)^{d}$ & $39(46.4)^{d}$ & $2(8.0)$ & $8(10.0)$ \\
\hline TLC (\% theoretical value) & $108.9 \pm 26.3$ & $106.8 \pm 31.5$ & $112.8 \pm 25.1$ & $102.3 \pm 17.0$ & $109.1 \pm 24.4$ \\
\hline Short-acting $\beta 2$-agonists, $n$ & 160 & 69 & 47 & 7 & 37 \\
\hline Long-acting $\beta 2$-agonists, $\mathrm{n}$ & 120 & 50 & 30 & 5 & 35 \\
\hline Long-acting anticholinergic, $n$ & 193 & 80 & 65 & 9 & 39 \\
\hline Bronchodilator associated with corticosteroids, $n$ & 145 & 58 & 40 & $\mathrm{II}$ & 36 \\
\hline Inhaled corticosteroids, $\mathrm{n}$ & 46 & 21 & 17 & 3 & 5 \\
\hline Oral corticosteroids, $\mathrm{n}$ & 61 & 38 & 12 & 2 & 9 \\
\hline
\end{tabular}

Notes: Data shown as $n$, mean \pm standard deviation, or $n$ (\%). aSignificantly different from NE $(p<0.05)$. ${ }^{b}$ Significantly different from CPAP and NIV ( $\left.p<0.05\right)$. ${ }^{\circ}$ Significantly different from all others groups $(p<0.05)$. ${ }^{d}$ Significantly different from CPAP and NE $(p<0.05)$. ${ }^{e}$ Significantly different from CPAP $(p<0.05)$.

Abbreviations: BMI, body mass index; CPAP, continuous positive airway pressure; $\mathrm{FEV}_{1}$, forced expiratory volume in I second; FVC, forced vital capacity; LTOT, long-term oxygen therapy; n, sample size; NE, no equipment; NIV, noninvasive ventilation; TLC, total lung capacity. 
Table 2 Comparison of exercise capacities, anxiety, depression and HRQoL at baseline and different times after home-based PR according to the medical equipment

\begin{tabular}{|c|c|c|c|c|c|}
\hline Test/questionnaire & $\begin{array}{l}\text { Medical } \\
\text { equipment }\end{array}$ & Baseline & Post PR & $\begin{array}{l}\text { After } \\
6 \text { months }\end{array}$ & $\begin{array}{l}\text { After } \\
12 \text { months }\end{array}$ \\
\hline \multirow[t]{4}{*}{ IOSTS (seconds) } & LTOT & $35.8 \pm 15.9^{\mathrm{a}, \mathrm{b}, \mathrm{c}}$ & $30.5 \pm 11.2^{\mathrm{a}, \mathrm{b}}$ & $34.0 \pm \mid 4.6^{\mathrm{a}, \mathrm{b}}$ & $34.4 \pm 16.5^{\mathrm{a}, \mathrm{b}}$ \\
\hline & NIV & $33.4 \pm 12.2^{c}$ & $28.1 \pm 10.3$ & $31.4 \pm 16.3$ & $30.8 \pm 11.8$ \\
\hline & CPAP & $24.0 \pm 7.9^{c}$ & $26.5 \pm 13.8$ & $26.4 \pm 16.0$ & $26.1 \pm 13.4$ \\
\hline & NE & $28.5 \pm 12.0^{c}$ & $23.6 \pm 9.6$ & $24.1 \pm 10.5$ & $23.9 \pm 11.0$ \\
\hline \multirow[t]{4}{*}{ TUG (seconds) } & LTOT & $13.0 \pm 10.2^{\mathrm{a}, \mathrm{c}}$ & $11.6 \pm 10.2^{\mathrm{a}}$ & $11.6 \pm 6.8^{\mathrm{a}}$ & $10.8 \pm 5.5^{\mathrm{a}}$ \\
\hline & NIV & $11.3 \pm 5.0^{c}$ & $10.5 \pm 9.2$ & $10.7 \pm 5.7$ & $10.3 \pm 4.2$ \\
\hline & CPAP & $8.9 \pm 4.6^{c}$ & $8.3 \pm 4.4$ & $8.5 \pm 5.9$ & $8.9 \pm 7.3$ \\
\hline & $\mathrm{NE}$ & $9.1 \pm 4.8^{c}$ & $7.4 \pm 2.9$ & $7.5 \pm 3.3$ & $7.5 \pm 3.8$ \\
\hline \multirow[t]{4}{*}{ 6MST (strokes) } & LTOT & $248 \pm 138^{a, b, d}$ & $315 \pm 156^{a, b}$ & $300 \pm 174^{a, b}$ & $342 \pm 195^{a, b}$ \\
\hline & NIV & $269 \pm 134^{d}$ & $372 \pm 159$ & $343 \pm 172$ & $410 \pm 137$ \\
\hline & CPAP & $432 \pm 171^{d}$ & $480 \pm 209$ & $486 \pm 255$ & $486 \pm 247$ \\
\hline & NE & $395 \pm 155^{d}$ & $444 \pm 155$ & $469 \pm 173$ & $486 \pm|7|$ \\
\hline HAD scale: anxiety & LTOT & $9.7 \pm 4.6^{d}$ & $8.7 \pm 4.3$ & $7.8 \pm 4.4$ & $8.1 \pm 4.7$ \\
\hline \multirow[t]{3}{*}{ state $(/ 2 \mathrm{I})$} & NIV & $9.6 \pm 4.6^{d}$ & $8.8 \pm 4.1$ & $8.0 \pm 4.6$ & $8.3 \pm 4.7$ \\
\hline & CPAP & $10.0 \pm 4.6^{d}$ & $8.1 \pm 4.4$ & $8.1 \pm 5.0$ & $8.4 \pm 5.3$ \\
\hline & NE & $9.6 \pm 4.5^{d}$ & $8.3 \pm 4.3$ & $7.3 \pm 4.3$ & $7.8 \pm 4.1$ \\
\hline HAD scale: & LTOT & $8.1 \pm 4.1^{d}$ & $6.7 \pm 4.3$ & $6.4 \pm 4.3$ & $6.8 \pm 4.7$ \\
\hline \multirow[t]{3}{*}{ depression state $(/ 2 \mathrm{I})$} & NIV & $7.9 \pm 3.9^{d}$ & $6.2 \pm 3.9$ & $5.9 \pm 3.6$ & $5.9 \pm 4.4$ \\
\hline & CPAP & $7.2 \pm 4.4^{\mathrm{d}}$ & $5.3 \pm 4.7$ & $5.1 \pm 4.7$ & $4.8 \pm 3.6$ \\
\hline & NE & $6.9 \pm 4.1^{d}$ & $5.4 \pm 3.7$ & $4.5 \pm 3.5$ & $4.9 \pm 3.7$ \\
\hline HAD scale: global & LTOT & $17.8 \pm 7.2^{\mathrm{d}}$ & $15.4 \pm 7.6^{\mathrm{e}}$ & $14.3 \pm 7.6$ & $14.9 \pm 8.1$ \\
\hline \multirow[t]{3}{*}{ score $(/ 42)$} & NIV & $17.2 \pm 7.0^{\mathrm{d}}$ & $15.0 \pm 7.1^{e}$ & $13.8 \pm 7.2$ & $14.2 \pm 7.8$ \\
\hline & CPAP & $16.9 \pm 7.5^{d}$ & $13.4 \pm 8.3^{e}$ & $|3.| \pm 8 . \mid$ & $13.2 \pm 7.8$ \\
\hline & $\mathrm{NE}$ & $16.4 \pm 7.7^{\mathrm{d}}$ & $13.7 \pm 6.9^{e}$ & II.8ะ6.7 & $12.7 \pm 6.4$ \\
\hline \multirow[t]{4}{*}{ MRF-28 (/I00) } & LTOT & $56.1 \pm 20.9^{d}$ & $47.9 \pm 22.0$ & $45.9 \pm 21.7$ & $50.0 \pm 22.4$ \\
\hline & NIV & $51.7 \pm 21.8^{d}$ & $44.7 \pm 23.4$ & $46.3 \pm 25.7$ & $43.4 \pm 25.5$ \\
\hline & CPAP & $40.3 \pm 20.6^{d}$ & $35.3 \pm 24.6$ & $36.9 \pm 26.1$ & $32.6 \pm 26.2$ \\
\hline & $\mathrm{NE}$ & $40.5 \pm 23.0^{d}$ & $32.7 \pm 20.8$ & $26.2 \pm 18.3$ & $30.0 \pm 20.9$ \\
\hline \multirow[t]{4}{*}{ VSRQ (/80) } & LTOT & $30.3 \pm 15.5^{d}$ & $37.3 \pm 16.7$ & $37.6 \pm 16.5$ & $34.1 \pm 14.8$ \\
\hline & NIV & $32.5 \pm 13.3^{d}$ & $39.5 \pm 15.7$ & $39.3 \pm 16.1$ & $39.3 \pm 16.7$ \\
\hline & CPAP & $31.4 \pm 14.8^{d}$ & $41.1 \pm 18.3$ & $38.8 \pm 16.7$ & $41.5 \pm 16.8$ \\
\hline & NE & $37.2 \pm 16.5^{d}$ & $42.7 \pm 16.8$ & $44.9 \pm 15.5$ & $43.4 \pm 15.5$ \\
\hline \multirow[t]{4}{*}{ VQII (/55) } & LTOT & $36.0 \pm 8.5^{d}$ & $32.4 \pm 8.8^{e}$ & $30.6 \pm 8.3$ & $33.3 \pm 8.9$ \\
\hline & NIV & $32.9 \pm 8.4^{\mathrm{d}}$ & $30.7 \pm 10.1^{\mathrm{e}}$ & $28.8 \pm 9.5$ & $28.8 \pm I I . I$ \\
\hline & CPAP & $33.0 \pm 6.8^{\mathrm{d}}$ & $29.9 \pm 10.0^{e}$ & $25.9 \pm 10.5$ & $27.7 \pm 7.9$ \\
\hline & NE & $31.2 \pm 9.2^{\mathrm{d}}$ & $27.1 \pm 9.4^{e}$ & $25.8 \pm 8.9$ & $27.2 \pm 9.5$ \\
\hline
\end{tabular}

Notes: aSignificantly different from NE ( $p<0.05)$. 'Significantly different from CPAP $(p<0.05)$. 'Significantly different compared to post PR ( $p<0.05)$. ${ }^{\mathrm{d} S i g n i f i c a n t l y ~ d i f f e r e n t}$ from all other times $(p<0.05)$. eSignificantly different compared to after 6 months $(p<0.05)$.

Abbreviations: 6MST, 6-minute stepper test; IOSTS, 10 times sit-to-stand test; CPAP, continuous positive airway pressure; HAD, Hospital Anxiety and Depression; HRQoL, health-related quality of life; LTOT, long-term oxygen therapy; MRF-28, Maugeri Foundation Respiratory Failure Questionnaire; NE, no equipment; NIV, noninvasive ventilation; PR, pulmonary rehabilitation; TUG, timed up-and-go test; VQII, COPD-specific HRQoL Questionnaire; VSRQ, Visual Simplified Respiratory Questionnaire.

which showed more responders in the NIV group compared with NE and LTOT groups (Figure 2).

\section{Discussion}

This study shows that a personalized and comprehensive home-based PR program including physical training, health education, psychological support, and motivational communication is feasible and effective in COPD patients regardless of their medical equipment, with significant short- and long-term improvement in exercise tolerance, anxiety and depression, and HRQoL.

At baseline, our results showed that BMI was significantly higher in the CPAP and NIV groups. This result is unsurprising since CPAP is the cornerstone treatment of OSAS, which is often associated with overweight. ${ }^{5}$ Moreover, NIV is required to treat hypoventilation, a condition observed in severe COPD or in the frequent association of COPD with OSAS (overlap syndrome) or obesity 


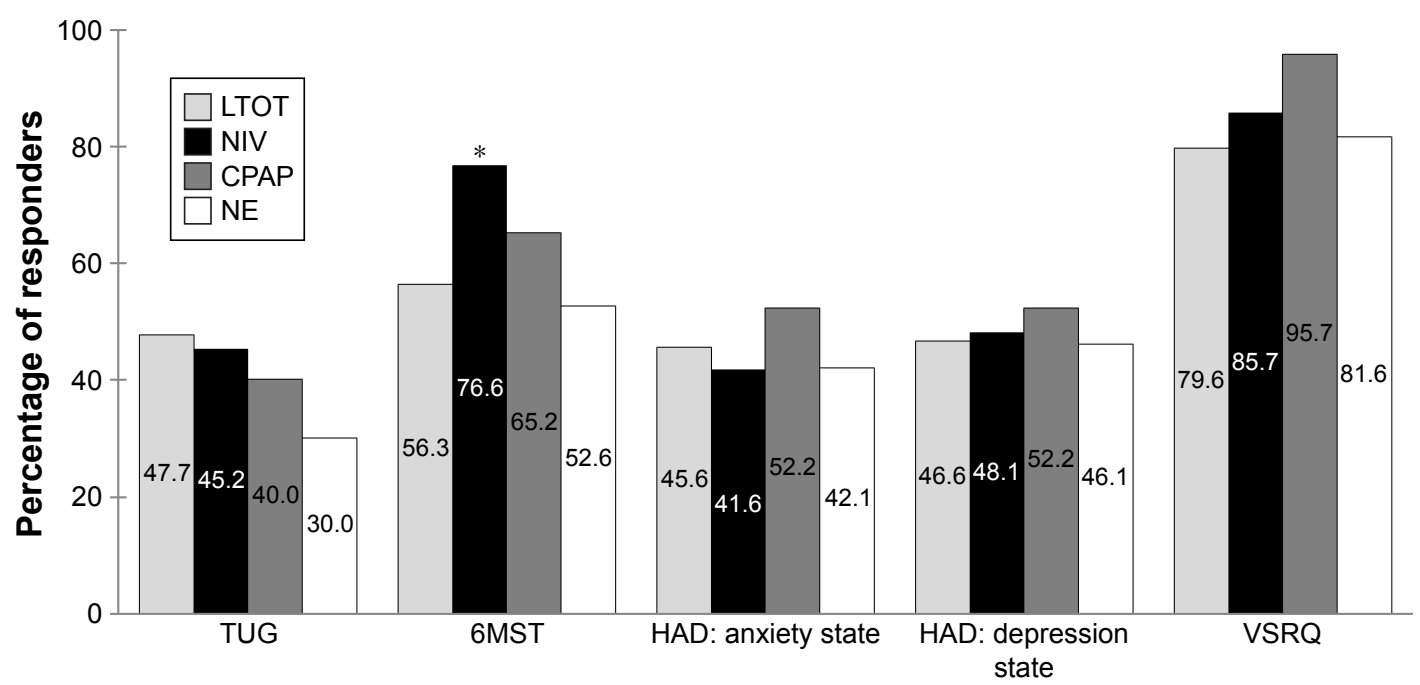

Figure 2 Percentage of patients who improved their exercise capacities, anxiety, depression, and health related quality of life.

Notes: Improvement was evaluated after 2 months of home-based pulmonary rehabilitation. Results are given according to patients' medical equipment. * $p<0.05$.

Abbreviations: 6MST, 6-minute stepper test; CPAP, continuous positive airway pressure; HAD, Hospital Anxiety and Depression; LTOT, long-term oxygen therapy; NE, no equipment; NIV, noninvasive ventilation; TUG, timed up-and-go test; VSRQ, Visual Simplified Respiratory Questionnaire.

hypoventilation syndrome. ${ }^{4}$ The number of $6 \mathrm{MST}$ responders was higher in the NIV group (and the CPAP group, but not significantly) compared with LTOT and NE groups (Figure 2). Recently, it was suggested that sleep quality might increase the amount of physical activity the next day and thus have a favorable impact on lifestyle. ${ }^{25}$ Moreover, it was previously shown in patients with obesity hypoventilation syndrome that exercise tolerance results increased with NIV and CPAP but more with NIV. ${ }^{26}$ Altogether, these findings suggest that the improvements in these groups may have been related to better nocturnal oxygenation and sleep quality. Another possible explanation is that patients with NIV and CPAP have higher lean mass than those with LTOT and $\mathrm{NE}$, who have lower BMI. It is not possible to confirm this hypothesis because we did not determine body composition, but complementary studies may be proposed.

In COPD patients with severe or very severe airflow limitation, LTOT is generally introduced for arterial hypoxemia with or without hypercapnia, while NIV is indicated for respiratory acidosis and/or hypercapnia or severe dyspnea with clinical signs suggestive of respiratory muscle fatigue, increased work of breathing, or both. ${ }^{4}$ Although our patients in the LTOT and NIV groups had more severe airflow limitation, the benefits of home-based PR were comparable to those of the patients in the CPAP and NE groups. Home-based PR programs have resulted in improvements in exercise tolerance, anxiety/depression, and/or HRQoL in COPD patients, regardless of age, sex, or even severity of airflow limitation. ${ }^{2,3,11,12}$ Conversely, Dias et $\mathrm{al}^{27}$ did not report these improvements. This divergence may be explained by methodological differences, as, for example, the number of training sessions per week was lower in their study in comparison with our protocol (ie, three vs five sessions per week). In addition, our individualized physical exercise program was adjusted to optimally correspond to each patient's daily real-life possibilities. Indeed, as this method has been shown to be valid and efficient, the patients themselves regulated exercise intensity from moderate symptoms of dyspnea or effort perception. ${ }^{28}$ Moreover, if a patient was unable to maintain 30 minutes of consecutive physical exercise, he or she was encouraged to perform several 10-minute sequences to reach this daily recommended exercise duration in the short term, medium term (6 months), and long term (12 months). ${ }^{29}$ This individualized exercise program thus seems efficient and feasible for COPD patients, especially those with severe or very severe airway obstruction. Last, our personalized and comprehensive PR program including health education, psychological support, and motivational communication probably enhanced the patients' intrinsic motivation to adopt certain health behaviors (eg, daily physical exercise, deambulation oxygen therapy if adapted) and to maintain these changes over the long term. ${ }^{10,30,31}$

An earlier study showed that very severe COPD patients with LTOT exhibited significantly greater improvements in (among others) physical exercise tolerance and HRQoL than patients without LTOT after an intensive 3-week full-day inpatient rehabilitation program (mean: $23 \pm 5$ days). ${ }^{7}$ The authors found that the improvements were more pronounced in those patients with worse baseline characteristics. These results are comparable to those of the present study, which 
required only one physical exercise session under the direct supervision of a team member (for 8 weeks). ${ }^{7}$ Indeed, patients in the LTOT and NIV groups had significantly greater improvements in $6 \mathrm{MST}(27.0 \%$ and $38.3 \%$, respectively) compared with the CPAP and NE patients $(11.1 \%$ and $12.4 \%$, respectively). It may therefore be recommended that patients with LTOT or NIV (who have greater airways obstruction severity) perform several 10-minute sequences of physical exercise to reach 30 minutes of exercise per day. ${ }^{29}$

Not all COPD patients benefit from PR to the same extent, but it is difficult to clearly identify those who will respond. Among the factors likely to improve with PR are the following: lower initial exercise capacity and greater ventilatory reserve, less ventilatory limitation to exercise and more reduced respiratory and peripheral muscle strength in patients with reduced exercise capacity, and $\mathrm{BMI} \geq 25 \mathrm{~kg} / \mathrm{m}^{2}$ or $\mathrm{PaO}_{2}<60 \mathrm{mmHg}$ (linked to greater deconditioning in obese patients) ${ }^{32-34}$ According to Altenburg et al, ${ }^{35}$ responders show hyperinflation, lower $\mathrm{FEV}_{1}$ (in percentage of theoretical value), lower quadriceps force, and lower exercise capacity. Furthermore, a recent meta-analysis demonstrated the shortterm effectiveness of inpatient, outpatient, and home- or community-based training programs lasting at least 4 weeks in improving functional capacity and HRQoL for stable, very severe COPD patients without major comorbidities. ${ }^{36}$ In addition, it was reported that comorbidities do not influence the response to PR. ${ }^{31}$ The current study confirms that homebased PR has numerous beneficial effects on COPD patients, independent of the number of comorbidities. Altogether, poor exercise capacity seems to be a good predictor of PR responders but not of comorbidities, although further studies must be conducted to determine other predictors.

The design of this study offered notable advantages in that it helped to better understand clinical practice in real life and to examine the long-term effects of home-based PR in COPD patients. Nevertheless, the design also had some limitations. Indeed, the analysis of this routine clinical practice was retrospective and observational and thus lacked the rigor of a randomized control trial. For example, the decision to perform home-based PR was dependent on the patient's personal preferences, thus meeting the needs of certain patients for various reasons (eg, difficulties getting around, lack of motivation for group rehabilitation and/or inpatient or outpatient centers, transport problems, distance from the nearest PR center, disruption of everyday habits, and program duration). ${ }^{12}$ Moreover, the patients of all four groups benefited from the PR program, which may indicate that medical equipment is not an efficient means for identifying responders to PR. Yet, we were unable to identify the types of management that would have been more efficient for specific groups because of the study design. This may also be considered as a limitation of our study as we cannot offer recommendations for improving the personalization of a PR program based on medical equipment. However, this seems to point to a strength of the current program, which adapts the intervention based on the initial evaluation and yields similar benefits regardless of baseline severity and comorbidities.

\section{Conclusion}

This retrospective, observational, routine clinical practice study reveals that home-based PR (including only one visit per week for 8 weeks) for COPD patients is feasible and safe and can significantly improve exercise tolerance, anxiety/ depression, and HRQoL over the short and long terms, especially for those with severe or very severe airways obstruction, regardless of their medical equipment (LTOT, NIV, CPAP). Home-based PR is an alternative to outpatient or inpatient rehabilitation and may make it easier for a greater number of patients to benefit from PR all over the world.

\section{Acknowledgments}

The authors would like to thank the rehabilitation team who managed the patients: G Tywoniuk, S Duriez, F Urbain, V Wauquier, and M Lambinet. The authors would also like to thank Adair, France Oxygène, Homeperf, LVL Medical, Orkyn, Santélys, SOS Oxygène, Sysmed, VitalAire, and the ARS Hauts-de-France for their support of the home-based PR program.

\section{Disclosure}

JM Grosbois received financial support from Adair, France Oxygène, Homeperf, LVL, Orkyn, Santélys, SOS Oxygène, Sysmed, VitalAire, and the ARS Hauts-de-France for homebased PR program. The other authors report no conflicts of interest in this work.

\section{References}

1. Barreiro E, Gea J. Respiratory and limb muscle dysfunction in COPD. COPD. 2015;12(4):413-426.

2. Spruit MA, Singh SJ, Garvey C, et al; ATS/ERS Task Force on Pulmonary Rehabilitation. An official American Thoracic Society/European Respiratory Society statement: key concepts and advances in pulmonary rehabilitation. Am J Respir Crit Care Med. 2013;188(8):e13-e64.

3. Liu X-L, Tan J-Y, Wang T, et al. Effectiveness of home-based pulmonary rehabilitation for patients with chronic obstructive pulmonary disease: a meta-analysis of randomized controlled trials. Rehabil Nurs. 2014;39(1):36-59.

4. Vogelmeier CF, Criner GJ, Martinez FJ, et al. Global strategy for the diagnosis, management, and prevention of chronic obstructive lung disease 2017 report. GOLD executive summary. Am J Respir Crit Care Med 2017;195(5):557-582. 
5. Kushida CA, Littner MR, Hirshkowitz M, et al; American Academy of Sleep Medicine. Practice parameters for the use of continuous and bilevel positive airway pressure devices to treat adult patients with sleep-related breathing disorders. Sleep. 2006;29(3):375-380.

6. Rizzi M, Grassi M, Pecis M, et al. A specific home care program improves the survival of patients with chronic obstructive pulmonary disease receiving long term oxygen therapy. Arch Phys Med Rehabil. 2009;90(3): 395-401.

7. Greulich T, Koczulla AR, Nell C, et al. Effect of a three-week inpatient rehabilitation program on 544 consecutive patients with very severe COPD: a retrospective analysis. Respiration. 2015;90(4):287-292.

8. Köhnlein T, Schönheit-Kenn U, Winterkamp S, Welte T, Kenn K. Noninvasive ventilation in pulmonary rehabilitation of COPD patients. Respir Med. 2009;103(9):1329-1336.

9. Wang T-Y, Lo Y-L, Lee K-Y, et al. Nocturnal CPAP improves walking capacity in COPD patients with obstructive sleep apnoea. Respir Res. 2013;14:66.

10. McCarthy B, Casey D, Devane D, Murphy K, Murphy E, Lacasse Y. Pulmonary rehabilitation for chronic obstructive pulmonary disease. Cochrane Database Syst Rev. 2015;2:CD003793.

11. Coquart JB, Grosbois JM, Olivier C, Bart F, Castres I, Wallaert B. Home-based neuromuscular electrical stimulation improves exercise tolerance and health-related quality of life in patients with COPD. Int J Chron Obstruct Pulmon Dis. 2016;11:1189-1197.

12. Grosbois J-M, Gicquello A, Langlois C, et al. Long-term evaluation of home-based pulmonary rehabilitation in patients with COPD. Int $J$ Chron Obstruct Pulmon Dis. 2015;10:2037-2044.

13. Borg GA. Psychophysical bases of perceived exertion. Med Sci Sports Exerc. 1982;14(5):377-381.

14. Borg GA. Perceived exertion as an indicator of somatic stress. Scand J Rehabil Med. 1970;2(2):92-98.

15. Netz Y, Ayalon M, Dunsky A, Alexander N. 'The multiple-sit-to-stand' field test for older adults: what does it measure? Gerontology. 2004; 50(3):121-126.

16. Mesquita R, Wilke S, Smid DE, et al. Measurement properties of the timed up \& go test in patients with COPD. Chron Respir Dis. Epub 2016 May 10.

17. Coquart JB, Lemaître F, Castres I, Saison S, Bart F, Grosbois JM. Reproducibility and sensitivity of the 6-minute stepper test in patients with COPD. COPD. 2015;12(5):533-538.

18. Grosbois JM, Riquier C, Chehere B, et al. Six-minute stepper test: a valid clinical exercise tolerance test for COPD patients. Int J Chron Obstruct Pulmon Dis. 2016;11:657-663.

19. Pichon R, Couturaud F, Mialon P, et al. Responsiveness and minimally important difference of the 6-minute stepper test in patients with chronic obstructive pulmonary disease. Respiration. 2016;91(5):367-373.

20. Zigmond AS, Snaith RP. The hospital anxiety and depression scale. Acta Psychiatr Scand. 1983;67(6):361-370.

21. Janssens J-P, Héritier-Praz A, Carone M, et al. Validity and reliability of a French version of the MRF-28 health-related quality of life questionnaire. Respiration. 2004;71(6):567-574.
22. Perez T, Arnould B, Grosbois J-M, et al; TIPHON Study Group. Validity, reliability, and responsiveness of a new short Visual Simplified Respiratory Questionnaire (VSRQ) for health-related quality of life assessment in chronic obstructive pulmonary disease. Int $J$ Chron Obstruct Pulmon Dis. 2009;4:9-18.

23. Ninot G, Soyez F, Préfaut C. A short questionnaire for the assessment of quality of life in patients with chronic obstructive pulmonary disease: psychometric properties of VQ11. Health Qual Life Outcomes. 2013;11:179.

24. Smid DE, Franssen FME, Houben-Wilke S, et al. Responsiveness and MCID estimates for CAT, CCQ, and HADS in patients with COPD undergoing pulmonary rehabilitation: a prospective analysis. $J \mathrm{Am} \mathrm{Med}$ Dir Assoc. 2017;18(1):53-58.

25. Spina G, Spruit MA, Alison J, et al. Analysis of nocturnal actigraphic sleep measures in patients with COPD and their association with daytime physical activity. Thorax. 2017;72(8):694-701.

26. Masa JF, Corral J, Alonso ML, et al; Spanish Sleep Network. Efficacy of different treatment alternatives for obesity hypoventilation syndrome: Pickwick study. Am J Respir Crit Care Med. 2015;192(1):86-95.

27. Dias FD, Sampaio LMM, da Silva GA, et al. Home-based pulmonary rehabilitation in patients with chronic obstructive pulmonary disease: a randomized clinical trial. Int J Chron Obstruct Pulmon Dis. 2013;8: $537-544$.

28. Zainuldin R, Mackey MG, Alison JA. Prescribing cycle exercise intensity using moderate symptom levels in chronic obstructive pulmonary disease. J Cardiopulm Rehabil Prev. 2016;36(3):195-202.

29. Donaire-Gonzalez D, Gimeno-Santos E, Balcells E, et al. Physical activity in COPD patients: patterns and bouts. Eur Respir J. 2013;42(4): 993-1002

30. Bourbeau J, Lavoie KL, Sedeno M. Comprehensive self-management strategies. Semin Respir Crit Care Med. 2015;36(4):630-638.

31. Spruit MA, Augustin IML, Vanfleteren LE, et al; CIRO+ Rehabilitation Network. Differential response to pulmonary rehabilitation in COPD: multidimensional profiling. Eur Respir J. 2015;46(6):1625-1635.

32. Zu Wallack RL, Patel K, Reardon JZ, Clark BA 3rd, Normandin EA. Predictors of improvement in the 12-minute walking distance following a six-week outpatient pulmonary rehabilitation program. Chest. 1991; 99(4):805-808

33. Troosters T, Gosselink R, Decramer M. Exercise training in COPD: how to distinguish responders from nonresponders. J Cardiopulm Rehabil. 2001;21(1):10-17.

34. Vagaggini B, Costa F, Antonelli S, et al. Clinical predictors of the efficacy of a pulmonary rehabilitation programme in patients with COPD. Respir Med. 2009;103(8):1224-1230.

35. Altenburg WA, de Greef MH, ten Hacken NH, Wempe JB. A better response in exercise capacity after pulmonary rehabilitation in more severe COPD patients. Respir Med. 2012;106(5):694-700.

36. Paneroni M, Simonelli C, Vitacca M, Ambrosino N. Aerobic exercise training in very severe chronic obstructive pulmonary disease: a systematic review and meta-analysis. Am J Phys Med Rehabil. 2017;96(8): $541-548$.
International Journal of COPD

\section{Publish your work in this journal}

The International Journal of COPD is an international, peer-reviewed journal of therapeutics and pharmacology focusing on concise rapid reporting of clinical studies and reviews in COPD. Special focus is given to the pathophysiological processes underlying the disease, intervention programs, patient focused education, and self management protocols.
Dovepress

This journal is indexed on PubMed Central, MedLine and CAS. The manuscript management system is completely online and includes a very quick and fair peer-review system, which is all easy to use. Visit http://www.dovepress.com/testimonials.php to read real quotes from published authors. 